\title{
ON NONLINEAR CONTRACTIONS
}

\author{
D. W. BOYD ${ }^{1}$ AND J. S. W. WONG ${ }^{2}$
}

Introduction. A well-known theorem of Banach states that if $X$ is a complete metric space and if $T$ is a mapping of $X$ into itself which satisfies

$$
\rho(T x, T y) \leqq k \rho(x, y),
$$

for some $k<1$ and all $x, y \in X$, then $T$ has a unique fixed point $x_{0}$, and the successive approximations $\left\{T^{n} x\right\}$ converge to $x_{0}$ for $x \in X$.

On the other hand, the condition

$$
\rho(T x, T y)<\rho(x, y)
$$

does not insure that $T$ has a fixed point.

In this paper, we investigate mappings which satisfy the following condition:

$$
\rho(T x, T y) \leqq \psi(\rho(x, y)),
$$

where $\psi$ is some function defined on the closure of the range of $\rho$.

In [3], Rakotch proved that if $\psi(t)=\alpha(t) t$, where $\alpha$ is decreasing and $\alpha(t)<1$ for $t>0$, then a mapping satisfying (3) has a unique fixed point $x_{0}$. It is an easy exercise to show that if $\psi(t)=\alpha(t) t$, where $\alpha$ is increasing, and $\alpha(t)<1$ for $t \geqq 0$, then the conclusion of Banach's theorem still holds. We shall show that one need only assume that $\psi(t)<t$ for $t>0$, together with a semicontinuity condition on $\psi$. For a metrically convex space, even this latter condition may be dropped.

A number of examples are given to show that the results do in fact improve upon those mentioned above.

We wish to thank the referee for suggesting the improved version of Theorem 1 which is presented in this paper.

We begin with some preliminary results on metrically convex spaces.

Definition 1 [1, p. 41]. A metric space $X$ is said to be metrically convex if for each $x, y \in X$, there is a $z \neq x, y$ for which $\rho(x, y)=\rho(x, z)$ $+\rho(z, y)$.

Presented to the Society, April 25, 1967; received by the editors July 25, 1967 and, in revised form, January $30,1968$.

1 Supported in part by NSF Grant GP 6111.

2 The work of the second named author was supported in part by University of Alberta General Research Fund no. 826. 
LEMMA 1 (MENGER). If $X$ is a complete metrically convex metric space, then for any $\alpha, 0<\alpha<1$, and any $x, y \in X$, there exists $z \in X$ such that

$$
\rho(x, z)=\alpha \rho(x, y) \text { and } \rho(z, y)=(1-\alpha) \rho(x, y) .
$$

Proof. See [1, p. 41, Theorem 14.1].

We shall denote the range of $\rho$ by $P$, and the closure of $P$ by $\bar{P}$, so $P=\{\rho(x, y) \mid x, y \in X\}$.

Note that, for a complete metrically convex space $X$, the set $P$ is convex and hence an interval $[0, b]$ or $[0, b)$, where $b \leqq \infty$. We shall use this notation in the following proofs.

Lemma 2. Suppose that $X$ is a complete metrically convex metric space and that $T: X \rightarrow X$ is a mapping which satisfies

$$
\rho(T x, T y) \leqq M \rho(x, y)
$$

for some constant $M<\infty$. Define the function $\phi:[0, b) \rightarrow[0, b]$ by

$$
\phi(t)=\sup \{\rho(T x, T y) \mid x, y \in X, \rho(x, y)=t\} .
$$

Then,

(a) $s>0, t>0$ and $s+t<b$ implies $\phi(s+t) \leqq \phi(s)+\phi(t)$; that is, $\phi$ is subadditive.

(b) $\phi$ is upper semicontinuous from the right on $[0, b)$.

Proof. (a) Let $\rho(x, y)=s+t$ where $x, y \in X$ and let $z \in X$ be such that $\rho(x, z)=s$, and $\rho(z, y)=t$ (possible by Lemma 1). Then

$$
\rho(T x, T y) \leqq \rho(T x, T z)+\rho(T z, T y) \leqq \phi(s)+\phi(t) .
$$

Taking the supremum of (6) over all $x, y \in X$ with $\rho(x, y)=s+t$ we obtain (a).

(b) From (a), if $t, t_{0}, t-t_{0}<b$ and $t>t_{0}$, then

$$
\phi(t) \leqq \phi\left(t-t_{0}\right)+\phi\left(t_{0}\right) \leqq M\left(t-t_{0}\right)+\phi\left(t_{0}\right),
$$

Thus, $\lim \sup _{t \rightarrow t_{0}+} \phi(t) \leqq \phi\left(t_{0}\right)$, proving (b).

Theorem 1. Let $X$ be a complete metric space, and let $T: X \rightarrow X$ satisfy (3), where $\psi: \bar{P} \rightarrow[0, \infty)$ is upper semicontinuous from the right on $\bar{P}$, and satisfies $\psi(t)<t$ for all $t \in \bar{P} \backslash\{0\}$. Then, $T$ has a unique fixed point $x_{0}$, and $T^{n} x \rightarrow x_{0}$ for each $x \in X$.

Proof. Given $x \in X$, define

$$
c_{n}=\rho\left(T^{n} x, T^{n-1} x\right) .
$$

Then, $c_{n}$ decreases to zero. For, by (3), $c_{n}$ is decreasing and hence has 
a limit $c$. But, if $c>0$, we have

$$
c_{n+1} \leqq \psi\left(c_{n}\right)
$$

so that

$$
c \leqq \lim \sup _{t \rightarrow c+} \psi(t) \leqq \psi(c),
$$

which is a contradiction.

Now, we show that for each $x \in X,\left\{T^{n} x\right\}$ is a Cauchy sequence. This will complete the proof, since the limit of this sequence is a fixed point of $T$ which is clearly unique. Suppose that $\left\{T^{n} x\right\}$ is not a Cauchy sequence. Then, there is an $\epsilon>0$ and sequences of integers $\{m(k)\},\{n(k)\}$, with $m(k)>n(k) \geqq k$, and such that

$$
d_{k}=\rho\left(T^{m} x, T^{n} x\right) \geqq \epsilon \quad \text { for } k=1,2, \cdots .
$$

We may assume that

$$
\rho\left(T^{m-1} x, T^{n} x\right)<\epsilon,
$$

by choosing $m(k)$ to be the smallest number exceeding $n(k)$ for which (10) holds. Recalling (7), we have

$$
d_{k} \leqq \rho\left(T^{m} x, T^{m-1} x\right)+\rho\left(T^{m-1} x, T^{n} x\right) \leqq c_{m}+\epsilon \leqq c_{k}+\epsilon .
$$

Hence, $d_{k} \rightarrow \epsilon+$, as $k \rightarrow \infty$.

But now,

$$
\begin{aligned}
d_{k}= & \rho\left(T^{m} x, T^{n} x\right) \leqq \rho\left(T^{m} x, T^{m+1} x\right)+\rho\left(T^{m+1} x, T^{n+1} x\right) \\
& +\rho\left(T^{n+1} x, T^{n} x\right) \leqq 2 c_{k}+\psi\left(\rho\left(T^{m} x, T^{n} x\right)\right)=2 c_{k}+\psi\left(d_{k}\right) .
\end{aligned}
$$

Thus, as $k \rightarrow \infty$ in (13), we obtain $\epsilon \leqq \psi(\epsilon)$, which is a contradiction for $\epsilon>0$.

TheOREM 2. Suppose that $X$ is a complete metrically convex metric space and that $T: X \rightarrow X$ satisfies (3), where $\psi: \bar{P} \rightarrow[0, \infty)$ satisfies $\psi(t)<t$ for all $t \in \bar{P} \backslash\{0\}$. Then, $T$ has a unique fixed point $x_{0}$, and $T^{n} x \rightarrow x_{0}$ for each $x \in X$.

Proof. Let $\phi(t)$ be defined as in Lemma 2 for $t \in[0, b)$. Then $\phi(t) \leqq \psi(t)$ for all $t \in[0, b)$. If $P=[0, b]$ with $b<\infty$, we define $\phi(b)$ $=\psi(b)$. Then,

$$
\rho(T x, T y) \leqq \phi(\rho(x, y)),
$$

for all $x, y \in X$, and $\phi(t) \leqq \psi(t)<t$ for $t \in \bar{P} \backslash\{0\}$. Also, by Lemma 2, $\phi$ is upper semicontinuous from the right on $[0, b)$. Thus, Theorem 1 applies to $T$, replacing $\psi$ by $\phi$ in that result. 
REMARKS. 1 . The continuity condition on $\psi$ cannot be omitted entirely from Theorem 1 as the following example shows:

Let $X=\left\{x_{n}=n \sqrt{ } 2+2^{n} \mid n=0, \pm 1, \pm 2, \cdots\right\}$, with the metric $\rho(x, y)=|x-y| . X$ is a closed subset of the reals so is complete. For each $p \in P, p \neq 0$, there is a unique pair $\left(x_{n}, x_{m}\right)$ such that $p=\rho\left(x_{n}, x_{m}\right)$. To see this, suppose that for some integers $j, k, m, n, j>k, m>n$ we have

Then

$$
\rho\left(x_{j}, x_{k}\right)=\rho\left(x_{m}, x_{n}\right) .
$$

$$
-(m-n-j+k) \sqrt{ } 2=2^{j}-2^{k}-2^{m}+2^{n} .
$$

Since the left member of (15) is irrational or zero, and the right member is rational, it follows that each member of (15) is zero. Thus, in particular $m-n=j-k=s$ say, and

$$
2^{n+s}-2^{n}=2^{k+s}-2^{k},
$$

which is possible only if $n=k$. Now, define $T$ by $T x_{n}=x_{n-1}$, and define $\psi$ on $P$ by

$$
\psi(p)=\left|x_{n-1}-x_{m-1}\right|, \quad \text { if } p=\left|x_{n}-x_{m}\right| .
$$

For $t \in \bar{P} \backslash P$, let $\psi(t)=0$.

Then, $\psi(t)<t$ for $t \in \bar{P} \backslash\{0\}$, and

$$
\rho(T x, T y)=\psi(\rho(x, y)),
$$

but $T$ has no fixed point.

Theorem 1 implies that there is no way to extend $\psi$ from $P$ to $\bar{P}$ to make $\psi$ upper semicontinuous from the right, and still have $\psi(t)<t$ for $t \in \bar{P} \backslash\{0\}$. This is easily seen directly for the point $\sqrt{ } 2 \in \bar{P} \backslash P$.

2 . If the condition that $\psi(t)<t$ is relaxed so that $\psi\left(t_{0}\right)=t_{0}$ for even one value of $t_{0}$ then the theorem may fail. In this case $T$ may have no fixed point or else more than one fixed point.

For a simple example, take $X=(-\infty,-1] \cup[1, \infty)$ with the absolute value metric. Let

and

$$
\begin{aligned}
T_{1} x & =\frac{1}{2}(x+1), & & x \geqq 1, \\
& =\frac{1}{2}(x-1), & & x \leqq-1,
\end{aligned}
$$

$$
T_{2} x=-T_{1} x .
$$

Then, $T_{1}$, and $T_{2}$ satisfy (3) with

$$
\begin{aligned}
\psi(t) & =\frac{1}{2} t, & & t<2, \\
& =\frac{1}{2} t+1, & & t \geqq 2 .
\end{aligned}
$$


The function $\psi$ satisfies all the conditions of Theorem 1 except that $\psi(2)=2 ; T_{1}$ has the two fixed points -1 and 1 , while $T_{2}$ has no fixed points.

3. We now present an example of a space $X$ and a mapping $T$ to show that Theorem 1 does improve the result of Rakotch. In fact, there can be no function $\alpha$, either decreasing or increasing, with $\alpha(t)<1$ for $t>0$, and such that

$$
\rho(T x, T y) \leqq \alpha(\rho(x, y)) \rho(x, y) .
$$

The space $X$ consists of the interval $[0,1]$ together with the integers $2,3,4, \cdots$. For the metric, let

$$
\begin{aligned}
\rho(x, y) & =|x-y| & & \text { if } x, y \in[0,1], \\
& =x+y & & \text { if one of } x, y \notin[0,1] .
\end{aligned}
$$

It is apparent that $(X, \rho)$ is a complete metric space once it is noticed that $(X, \rho)$ is isometric to a closed subset $Y$ of the space $l^{1}$ of absolutely summable sequences. The set $Y$ consists of the sequences $(x, 0,0, \cdots)$ for $x \in[0,1]$ together with the sequences with $m$ in the $m$ th coordinate place and zeros elsewhere $(m=2,3, \cdots)$.

Define the mapping $T: X \rightarrow X$ by

$$
\begin{aligned}
T x & =x-\frac{1}{2} x^{2} & & \text { if } x \in[0,1], \\
& =x-1 & & \text { if } x=2,3, \cdots .
\end{aligned}
$$

Then, for $x, y \in[0,1]$ with $x-y=t>0$,

$$
\rho(T x, T y)=(x-y)\left(1-\frac{1}{2}(x+y)\right) \leqq t\left(1-\frac{1}{2} t\right)
$$

and, if $x \in\{2,3,4, \cdots\}$ with $x>y$, then

$$
\rho(T x, T y)=T x+T y<x-1+y=\rho(x, y)-1 .
$$

Thus, if we define $\psi$ by

$$
\begin{aligned}
\psi(t) & =t-\frac{1}{2} t^{2}, & & 0 \leqq t \leqq 1, \\
& =t-1, & & 1<t<\infty,
\end{aligned}
$$

then $\psi$ is upper semicontinuous from the right on $[0, \infty), \psi(t)<t$ for all $t>0$, and (3) holds.

However, as $n \rightarrow \infty, \rho(T n, 0) / \rho(n, 0) \rightarrow 1$, so there can be no decreasing function $\alpha$ with $\alpha(t)<1$ for $t>0$, and for which (20) holds. Furthermore, $\rho(T x, 0) / \rho(x, 0) \rightarrow 1$ as $x \rightarrow 0$, so there is no increasing function $\alpha$ with $\alpha(t)<1$ for $t>0$, and for which (20) holds.

4. Although the previous example shows that Theorem 1 is in fact more general than the result due to Rakotch, one would prefer an 
example in which the space $X$ is metrically convex. The following proposition shows that it would be difficult to obtain such an example.

Proposition. Let $X$ and $T$ satisfy the conditions of Theorem 2, and let $\phi$ be defined as in Lemma 2. Suppose, for any $c$ and $d$ satisfying $0<c<d<\infty$ that

$$
\sup _{c \leqq l \leqq d} \phi(t) / t<1 .
$$

Then, there is a decreasing function $\alpha$ with $\alpha(t)<1$ for all $t>0$, such that $\rho(T x, T y) \leqq \alpha(\rho(x, y)) \rho(x, y)$.

In particular, if $\psi$ is increasing, or if $\phi$ is upper semicontinuous from the left then such an $\alpha$ exists.

Proof. We suppose that $P=[0, \infty)$. The case where $P=[0, b]$ or $[0, b)$ with $b<\infty$ is even easier to handle.

From Lemma 2, $\phi$ is subadditive, so by Theorem 7.6.2 of [2], $\beta=\lim _{t \rightarrow \infty} \phi(t) / t$ exists and equals inf $t>0 \phi(t) / t$. But, $\phi(t)<t$ for $t>0$, so $\beta<1$. Hence, there is a number $d$ such that

$$
\phi(t) / t<\frac{1}{2}(1+\beta)<1 \quad \text { for } t \geqq d .
$$

Now, let us define $\alpha$ as follows:

$$
\alpha(t)=\sup _{s \geqq \iota} \phi(s) / s .
$$

Then $\alpha$ is decreasing, and furthermore $\alpha(t)<1$ for $t>0$ because of (24) and (25).

To prove the remainder of the assertions note that if $\psi$ is increasing and we define

$$
\phi^{*}(t)=\sup _{0 \leqq s \leqq} \phi(t),
$$

then $\phi^{*}(t) \leqq \psi(t)$ for all $t>0$, and $\phi^{*}$ is subadditive, increasing, and continuous at 0 . Thus, for $t>0, h>0, t-h>0$,

$$
\phi^{*}(t-h) \leqq \phi^{*}(t) \leqq \phi^{*}(t-h)+\phi^{*}(h),
$$

and

$$
\phi^{*}(t) \leqq \phi^{*}(t+h) \leqq \phi^{*}(t)+\phi^{*}(h) .
$$

Letting $h \rightarrow 0$ we see that $\phi^{*}$ is continuous for all $t \geqq 0$, and thus certainly

$$
\sup _{t \leqq t \leqq d} \phi(t) / t \leqq \max _{t \leq ı \leq d} \phi^{*}(t) / t<1
$$


The other assertion is handled similarly.

The authors would welcome an example of a function $\phi$ which is subadditive, positive, for which $\phi(t)<t$ for all $t>0$, and such that (24) does not hold.

ADDED IN PROOF. We note that the upper semicontinuity of the function $\psi$ may indeed be replaced by the weaker assumption that $\limsup _{s \rightarrow t+}+(s)<t$ for $t \in P$, (cf. (9)).

\section{REFERENCES}

1. L. E. Blumenthal, Theory and application of distance geometry, Clarendon Press, Oxford, 1953.

2. E. Hille and R. S. Phillips, Functional analysis and semi-groups, Amer. Math. Soc. Colloq. Publ., Vol. 31, Amer. Math. Soc., Providence, R. I., 1957.

3. E. Rakotch, $A$ note on contractive mappings, Proc. Amer. Math. Soc. 13 (1962), 459-465.

University of Alberta, California Institute of Technology, University of Wisconsin and Carnegie-Mellon University 